\title{
Philosophiques
}

\section{Réponse à mes critiques}

\section{John H. McDowell}

Volume 36, numéro 1, printemps 2009

L'idéalisme britannique

URI : https://id.erudit.org/iderudit/038020ar

DOI : https://doi.org/10.7202/038020ar

Aller au sommaire du numéro

Éditeur(s)

Société de philosophie du Québec

ISSN

0316-2923 (imprimé)

1492-1391 (numérique)

Découvrir la revue

Citer ce document

McDowell, J. H. (2009). Réponse à mes critiques. Philosophiques, 36(1), 221-233.

https://doi.org/10.7202/038020ar d'utilisation que vous pouvez consulter en ligne.

https://apropos.erudit.org/fr/usagers/politique-dutilisation/ 


\title{
Réponse à mes critiques"
}

\author{
JOHN H. MCDOWELL \\ Université de Pittsburgh
}

\section{Réponse à David Davies}

Davies introduit la question qui l'intéresse - en quoi ma conception de l'expérience contribue à rendre intelligible la redevabilité de notre pensée au monde - en rappelant à ses lecteurs les attaques, notamment celles de Robert Brandom et de Donald Davidson, contre l'idée même que l'expérience peut être pertinente pour la justification - en opposition à la simple étiologie - des jugements perceptuels. Il écrit dans ce contexte: "Placer l'expérience à l'intérieur de l'espace des raisons semble simplement être une façon de donner une nouvelle étiquette à nos jugements sensoriels plutôt que de caractériser l'engagement envers le monde, engagement à propos duquel sont émis de tels jugements. Insister sur le fait que l'expérience se trouve à l'intérieur de l'espace des raisons ne répond pas à la question de savoir comment elle peut impliquer le monde dans son contenu tel que l'exige [l'empirisme minimal].» (p. 197)

Je me demande à quel point il veut que nous prenions au sérieux cette introduction au sujet. Il est vrai qu'à quelqu'un qui ne peut voir comment autre chose que des jugements peut avoir le type de contenu pertinent, il semblera que dans l'explication que j'offre de l'expérience, je ne puisse qu'appliquer l'étiquette d' "expérience » à certains jugements. Mais, de mon point de vue, cette idée ne fait que refléter une simple tâche aveugle. Elle passe à côté de la possibilité sur laquelle j'insiste, à savoir que les mêmes capacités conceptuelles qui sont exercées dans des jugements peuvent également être actualisées dans la réceptivité sensorielle. Placer l'expérience dans l'espace des raisons n'est rien de plus qu'affirmer que cette possibilité est actuelle dans le cas de l'expérience perceptuelle des animaux rationnels. Dans le contexte qui nous intéresse ici, cela conduit à affirmer que les expériences perceptuelles des animaux rationnels ont le même type de contenu que les jugements ${ }^{1}$. Et le rôle que joue l'idée de la réceptivité sensorielle dans l'explication de l'expérience que je propose fait en sorte que cette affirmation ne revient pas simplement à appliquer l'étiquette d' «expérience » à des objets qui sont

* J'exprime ma reconnaissance à mes commentateurs pour s'être donné autant de peine à lire mon ouvrage, et je remercie le directeur de la revue de m'avoir donné l'occasion de prendre part à cette disputatio.

1. Je dis «dans le contexte qui nous intéresse ici » parce que je fais abstraction ici de l'idée à laquelle je n'ai fait attention que depuis que j'ai écrit L'esprit et le monde, à savoir que le contenu expérientiel est à concevoir davantage comme étant intuitif plutôt que propositionnel. Voir ma réponse à Jérôme Dokic pour de plus amples remarques sur ce point. 
véritablement des jugements. En fait, le rôle de la réceptivité sensorielle dans cette explication assure que les expériences peuvent être conçues comme des prises en charge de l'état des choses, ce qui est l'élément essentiel de mon explication de la manière par laquelle les expériences médiatisent la redevabilité au monde.

Supposons que quelqu'un soit prêt à soutenir que les expériences sont à l'intérieur de l'espace des raisons puisqu'elles ont le même type de contenu que celui des jugements. Je ne vois pas pourquoi une telle personne ne trouverait pas de réponse à la question de savoir comment l'expérience en particulier peut impliquer le monde dans son contenu, contrairement à ce que suggère Davies dans la deuxième phrase que j'ai citée de lui. Après tout, l'effet produit par l'affirmation est de créditer l'expérience de la même orientation vers le monde qu'ont les jugements. Peut-être que Davies envisage ici quelqu'un qui trouve déroutante cette orientation vers le monde en général, et non l'orientation vers l'expérience en particulier. Mais ce que je propose, c'est précisément que nous pouvons améliorer notre compréhension de l'orientation vers le monde prise en général, possédée comme elle l'est également par les expériences et les jugements, en l'expliquant dans un cas comme dans l'autre en termes d'actualisation de capacités conceptuelles et en mettant l'accent sur le fait que, dans le cas des expériences, l'actualisation se produit dans la réceptivité sensorielle.

D'une manière qui me semble étrange, Davies lui-même se distancie de l'idée que je vois le monde comme étant constitué de faits considérés comme des sens frégéens. Il n'introduit l'idée que comme une interprétation que certains lecteurs ont appliquée à certains passages de mon livre. Mais cette précaution n'était pas nécessaire. J'ai voulu être assez explicite dans mon livre sur le fait que je travaille avec l'idée que le monde est tout ce qui est le cas, ainsi qu'avec l'idée que quelque chose qui est le cas est quelque chose qu'on peut véridiquement penser comme étant le cas — autrement dit: la pensée, dans un sens semblable à celui de Frege, qui est le contenu d'un acte possible de penser vrai.

Davies construit la portion positive de son article en partant de deux types d'inquiétudes qu'on peut avoir sur cette question.

La première est l'idée que le portrait que je dresse ne rend pas compte des faits «en tant qu'états du monde rendant vraies des pensées vraies». Dans la version de cette inquiétude qu'on retrouve chez Julian Dodd, je devrais confondre les éléments du monde en mon sens - des choses dont on peut véridiquement penser qu'elles sont le cas - avec des faits dans un sens différent, selon lequel ils peuvent être des vérifacteurs.

La seconde est une inquiétude sur la compréhension interculturelle, formulée par Michael Friedman dans les termes d'une soi-disant menace du relativisme culturel ou linguistique: une menace selon laquelle je verrais différentes traditions culturelles occuper des espaces de raison différents, chacun constituant son propre monde. 
Contre la première inquiétude, j'insiste sur le fait que les éléments du monde pris en mon sens peuvent parfaitement être pris pour des vérifacteurs. Comme je le remarque dans ma réponse à Jérôme Dokic (qui a soulevé une version de cette inquiétude) on peut introduire des vérifacteurs au moyen d'un certain type de nominalisation: ce serait vrai de penser que le temps est ensoleillé aujourd'hui (imaginons un contexte d'énonciation convenable) en vertu de l'ensoleillement du temps aujourd'bui. Nous pouvons comprendre cette nominalisation comme référant à quelque chose dont on peut penser que c'est le cas - que le temps est ensoleillé aujourd'hui d'une manière qui signale qu'on peut véridiquement penser que c'est le cas. Ainsi, la nominalisation peut être comprise comme se référant à un élément du monde pris en mon sens. Mais l'aspect naturel de la locution «en vertu de " montre qu'il n'y a pas de problème à concevoir la nominalisation comme se référant à un vérifacteur.

La deuxième inquiétude, au moins dans la version de Friedman, n'arrive pas à vraiment porter attention à l'idée qui est sa cible. L'idée est que le monde est tout ce qui est le cas (et bien sûr seulement ce qui est le cas; les éléments du monde n'incluent pas des choses qui ne sont pas le cas). L'idée n'est pas que le monde de telle-ou-telle culture est tout ce que les membres de cette culture considèrent être le cas. ( $\mathrm{Si}$ on fait sens de la notion d'un monde de telle-ou-telle culture, c'est uniquement dans un type de contexte plutôt différent.) Il ne fait aucun doute que la compréhension interculturelle peut être ardue. Mais il n'est pas impossible d'arriver provisoirement à la conclusion que quelque chose qui est considéré comme étant le cas dans une culture étrangère n'est pas le cas, et ainsi n'est pas un élément du monde. Et cela bien sûr de notre point de vue, ce qui fait ressortir une raison pour le caractère provisoire de telles conclusions; lorsque nous tentons de comprendre des cultures étrangères, nous devrions rester ouverts à la possibilité que notre point de vue - et, de manière correspondante, notre vision du monde - puisse s'améliorer en apprenant des autres. C'est une confusion de penser qu'il y a là une ouverture au relativisme, une tendance à nous faire perdre prise sur l'idée $d u$ monde, sur l'état des choses (ce qui ne doit absolument pas être identifié avec l'état des choses pour nous). Le monde par rapport auquel notre vision du monde tend à être une vision est tout ce qui est le cas. Il est partie prenante de la manière dont nous comprenons cette visée que quelques-unes des choses qui sont le cas ne font pas partie de nos connaissances (bien qu'elles soient peut-être connues des autres, et que quelques-unes des choses que nous pensons être le cas ne sont pas le cas. Le monde est constitué par l'état actuel des choses. Il nous incombe de faire de notre mieux pour le découvrir. Et cela signifie également faire de notre mieux pour nous assurer que ce que nous considérons être des manières de le faire est effectivement ce que nous pensons qu'elles sont — c'est-àdire de nous assurer que notre conception de la structure des régions concernées de l'espace des raisons est correcte. 
Dans ces brèves remarques, j'ai un peu négligé les deux inquiétudes que Davies présente comme étant les motivations de son article. Je ne crois pas qu'elles mènent à grand chose. On comprendra donc que je soupçonne le «modèle dual de l'expérience", que Davies propose d'extraire de mon ouvrage, d'être un dispositif superflu, du moins pour l'usage en question ici. Et je suis de toute façon peu disposé à accepter ce que Davies suggère sous la rubrique du modèle dual.

Dans la mesure où le modèle dual ne fait que déclarer que la doctrine frégéenne inclut la Bedeutung ainsi que le Sinn, il ne me pose bien sûr aucun problème. Le monde, compris comme tout ce qui est le cas, a pour éléments les membres d'un sous-ensemble des pensées frégéennes: les pensées vraies. Les pensées frégéennes sont des sens et n'ont que des sens comme parties constituantes. Mais ces sens, qui sont les parties constituantes, incluent par exemple les modes de présentation des objets. Ainsi, même si les objets ne sont pas des parties constituantes des pensées, et donc ne sont pas des parties constituantes du monde compris comme tout ce qui est le cas, ils ne font pas défaut dans l'image d'ensemble selon laquelle, comme je le dis dans mon livre, l'acte de penser et de faire une expérience sont en contact avec le monde dans le domaine du sens. Ce type de considération peut effectivement nous aider à désamorcer le type d'inquiétude qu'on retrouve dans l'idée de Dodd selon laquelle j'aurais besoin d'un autre genre de choses qui serviraient de vérifacteurs - bien que le désamorçage fonctionne en s'assurant que les objets et autres Bedeutungen d'expressions infraphrastiques ne fassent pas défaut, et non en incluant des «états du monde» dans le domaine de la Bedeutung. Les Bedeutungen d'expressions dont le sens est constitué de pensées complètes ne sont pas des états du monde mais bien des valeurs de vérité, et il n'existe que deux de celles-ci (je reviendrai plus loin sur les états du monde).

Je ne crois pas que la distinction Sinn-Bedeutung, ou la distinction afférente entre les choses individuées extensionnellement et les choses qui ne le sont pas, ait un rapport particulier à la soi-disant inquiétude d'une ouverture vers le relativisme. Les choses qui sont le cas - les choses dont on peut véridiquement penser qu'elles sont le cas — ne sont absolument pas individuées extensionnellement. Même si chacun des actes d'une paire d'actes de penser implique, supposons, d'attribuer la même propriété au même objet, on sait qu'on ne montre pas de cette manière qu'ils ont la même pensée frégéenne comme contenu. Si, par exemple, l'objet figure dans ces deux actes de penser sous deux modes différents de présentation, les pensées sont différentes (c'est ainsi que Frege introduit sa distinction entre Sinn et Bedeutung). Et, de manière correspondante, si deux tels actes de penser sont vrais, les faits - les éléments du monde - en vertu desquels ils sont vrais sont alors différents. Il n'y a aucune ouverture au relativisme lorsqu'on reconnaît que les faits diffèrent. Il n'y a aucun renvoi, dans la doctrine de Frege, à une idée d'états du monde selon lesquels les expériences dont les 
contenus sont donnés par deux telles pensées sont, comme Davies semble le suggérer, des expériences du même état du monde, présenté différemment dans les deux expériences. (Ce ne serait pas simplement une autre application de la distinction Sinn-Bedeutung.) Le problème avec l'inquiétude de Friedman n'est pas qu'elle passe à côté de la possibilité d'élaborer l'idée d'un monde commun en termes d'états du monde conçus de cette manière, possédés comme des objets de l'expérience et du jugement entre des sujets dont les expériences et les jugements divergent selon l'aspect non extensionnel caractéristique des pensées frégéennes. Le point sur lequel l'inquiétude de Friedman ne réussit pas à mettre le poids nécessaire peut être formulé entièrement au niveau du Sinn, entièrement par des éléments qui, étant des pensées frégéennes, ne sont pas individués extensionnellement. Ce qui disperse l'inquiétude est que le monde visé par une vision du monde est tout ce qui est le cas (c'est-à-dire tous les membres de cette espèce individuée non extensionnellement qui ont le vrai pour valeur de vérité), et non pas tout ce qui est considéré comme étant le cas dans la vision du monde en question.

\section{Réponse à Jérôme Dokic}

Donald Davidson a exprimé une inquiétude relativement aux expériences perceptuelles prises comme intermédiaires entre les sujets percevants et le monde - des intermédiaires qu'on ne peut obliger à dire la vérité. Dans Mind and World, j'ai affirmé que cette inquiétude est neutralisée par ma conception de l'expérience perceptuelle. Selon ma conception, l'expérience perceptuelle n'est pas un intermédiaire, mais elle nous ouvre directement au monde lui-même. Dokic croit que ma réponse à Davidson ne peut fonctionner, et plus généralement que ma conception de la manière par laquelle l'expérience perceptuelle nous ouvre au monde ne peut fonctionner non plus. Sa motivation repose sur le fait qu'il y a selon lui une bipolarité caractérisant le contenu perceptuel comme tel, indépendamment de savoir si elle est conçue de la manière que je recommande dans le texte de Mind and World — c'est-à-dire un contenu propositionnel comme contenu potentiel de jugements - ou de la manière que j'ai esquissée brièvement dans l'édition française, c'est-à-dire comme contenu intuitif.

Je crois que cela est complètement erroné.

Commençons par le contenu intuitif. Une intuition peut avoir un contenu en vertu du fait qu'elle est une actualisation, avec un certain mode d'unité, des capacités conceptuelles dont le contenu peut être exprimé par « rouge » et «cube », pour prendre un exemple simple. En vertu du fait qu'il a un contenu du type pertinent (évidemment, un cas actuel serait plus complexe et plus spécifique), une telle intuition rendrait un cube rouge perceptuellement présent à un sujet. Et il n'y a ici aucune place pour aucun analogon de la bipolarité. Il est vrai qu'une expérience peut sembler rendre un cube rouge perceptuellement présent à un sujet sans vraiment le faire. C'est-à-dire qu'une expérience pourrait sembler avoir le type de contenu qui caractéri- 
serait une intuition d'un cube rouge, mais sans vraiment avoir ce type de contenu. Si une expérience a vraiment ce type de contenu, elle rend un cube rouge perceptuellement présent à son sujet. Il n'y a pas d'autre pôle capable d'être occupé par un contenu actuel de ce type - en opposition à un semblant de contenu de ce type - qui serait analogue à la fausseté dans le cas du contenu propositionnel.

Je choisis ici la première corne du dilemme que présente Dokic à mon sujet relativement au contenu intuitif: le contenu intuitif est un type de contenu différent du contenu propositionnel, ce n'est pas le même type de contenu vu différemment. Il est vrai que si nous concevons l'expérience comme comprenant les intuitions, l'épistémologie des jugements perceptuels, ne peut prendre exactement la forme qu'elle prend dans le portrait que j'ai dressé dans Mind and World, selon lequel les jugements perceptuels héritent directement leur contenu du contenu des expériences sur lesquelles ils sont basés, et selon lequel ils sont des jugements de connaissance en vertu du fait que les expériences révèlent que les choses sont telles qu'elles sont jugées dans ces jugements. Mais l'épistémologie du contenu intuitif est seulement un peu plus complexe. Le mode d'unité avec lequel ces capacités conceptuelles sont actualisées, dans une intuition comme celle que j'ai considérée plus haut, est une contrepartie, dans le domaine des unités intuitives, d'un mode d'unité dans le domaine des unités judicatives: à savoir le mode d'unité avec lequel ces mêmes capacités conceptuelles peuvent être exercées dans un jugement pouvant être exprimé par "il y a un cube rouge là-bas", par exemple. Et il est facile de voir comment le fait d'avoir un cube rouge perceptuellement présent - dans une expérience partiellement constituée par une actualisation de ces capacités conceptuelles avec un mode convenable d'unité intuitive - peut nous permettre d'émettre un jugement de connaissance dans lequel les mêmes capacités conceptuelles sont exercées avec le mode de l'unité judicative en contrepartie. Le dilemme de Dokic ne présente aucune menace ${ }^{2}$.

Toutefois, dans le cas présent, le contenu intuitif n'est pas ce qui est vraiment important. Je peux en rester à la conception de l'expérience perceptuelle des sujets rationnels que j'ai suggérée dans Mind and World, c'est-àdire avec un contenu propositionnel. Il y a en effet un sens dans lequel le contenu propositionnel est essentiellement bipolaire, mais Dokic a tort de penser que cela pose problème à la conception de l'ouverture au monde que j'ai suggérée. Ce n'était pas en tentant d'éviter une telle difficulté que j'ai suggéré une conception du contenu expérientiel comme contenu intuitif. Je

2. Dans la préface à l'édition française, je ne fais rien de plus qu'esquisser la compréhension du contenu intuitif avec lequel je travaille ici. Pour une plus ample discussion, voir mon article «Avoiding the Myth of the Given ", reproduit depuis peu dans mon ouvrage Having the World in View: Essays on Kant, Hegel and Sellars (Cambridge, Mass., Harvard University Press). Dans «Intentionality as a Relation », publié dans le même volume, j'élabore l'idée que quand on semble jouir d'un contenu intuitif, on ne jouit pas vraiment d'un tel contenu. 
crois que l'article de Dokic trahit une incompréhension de la signification de la bipolarité essentielle du contenu propositionnel et qu'il ne parvient pas à apprécier les ressources à la disposition d'une conception, frégéenne en substance, des pensées, des faits et du monde.

Bien sûr, Dokic a raison lorsqu'il dit que le contenu propositionnel n'est pas bipolaire sous une description. La capacité d'être vrai ou faux est essentielle au genre de choses que sont les propositions. Mais il sous-estime l'importance du fait que si on décrit une proposition comme ce que quelqu'un, par exemple, considère être le cas, on exclut ainsi le pôle de la fausseté. Concédant ce fait, Dokic écrit: "Quand un contenu est celui d'une expérience perceptive, le pôle de la fausseté est en effet exclu, de sorte que ce qui est perçu est un fait. » Et de poursuivre: "Toutefois, contrairement à ce que suppose McDowell, l'exclusion de ce pôle de facto ne signifie pas pour autant que le contenu, même en tant qu'il est perçu, cesse d'être bipolaire. » Mais le sens en lequel cela est correct n'affecte pas les prospects d'une conception globalement frégéenne. Certes, le fait qu'un contenu soit perçu comme étant le cas n'exclut pas qu'il appartienne à une sorte de choses dont il est essentiel que leurs membres puissent être vrais ou faux. Cela se reflète dans le fait que ce contenu est quelque chose qui pourrait avoir été faux. Mais toute possibilité actuelle d'un pôle de la fausseté est exclue pour lui. Sa capacité, qua propositionnelle, à être faux n'est pas actualisée. On ne peut le créditer de la potentialité d'être faux sans abandonner la supposition qu'il est perçu comme étant le cas.

Il en va de même pour une proposition qu'on considère non pas comme perçue, mais simplement comme étant le cas. Elle est du coup considérée comme quelque chose dont la capacité - qua propositionnelle - à être fausse n'est pas actualisée. Que cela aurait pu être faux, ce qui est tout ce que à quoi correspond la bipolarité ici, n'affecte pas la possibilité d'exploiter l'exclusion de facto du pôle de fausseté dans une conception frégéenne des faits, et dans une glosse frégéenne (et dans cette mesure non tractarienne) sur la conception tractarienne du monde comme tout ce qui est le cas.

Dokic pense qu'il découle de la bipolarité de la pensée que les vérifacteurs doivent être des entités d'un type différent de celui des pensées. Je crois qu'il y a là une confusion modale. Bien sûr, il y a un sens dans lequel un vérifacteur doit ne pas être bipolaire. Mais, si on la comprend correctement, cette exigence peut être satisfaite par un genre d'objets dont les membres sont bipolaires, en ce que les objets de ce genre ont la capacité d'être vrais ou faux - pourvu que dans le cas de l'objet en question la capacité à être faux ne soit pas actualisée, comme je l'ai déjà souligné.

Dans les environs de la remarque de Mark Johnston citée par Dokic dans ce contexte, ce qui est correct, c'est que la réalité concrète ne consiste pas en choses qui pourraient être fausses. Mais un contenu dont la capacité à être faux n'est pas actualisée n'est pas une chose qui pourrait être fausse. C'est une chose qui aurait pu être fausse. Et, contrairement à ce que dit 
Johnston, il n'y a aucun problème à supposer que la réalité concrète consiste en choses qui auraient pu être fausses. À condition que nous focalisions sur les instances de telles choses dont la capacité à être fausses n'est pas actualisée, celles-ci peuvent faire tout le travail pour lequel on pourrait vouloir invoquer des vérifacteurs ${ }^{3}$.

On ne doit pas donner trop de poids à l'ontologie qui semble ici implicite. Parler de vérifacteurs comme d'objets de telle et telle sorte n'est rien de plus qu'une manière utile de donner une expression générale à des pensées telles que celle-ci (imaginons un contexte d'énonciation approprié): il serait vrai de penser qu'il y a encore du café dans la tasse de Dokic parce qu'il y $a$ encore du café dans la tasse de Dokic. Nous pouvons explicitement ajouter un vérifacteur à notre ontologie en reformulant la phrase à l'aide d'une nominalisation: il serait vrai de penser qu'il y a encore du café dans la tasse de Dokic en vertu du fait qu'il y a encore du café dans la tasse de Dokic. Dans le genre d'occasion que je me suis imaginée, la nominalisation que j'ai mise en italique se rapporte certes à un objet — pour mettre les choses dans un registre ontologique, d'un genre dont les membres qui ont la capacité d'être vrais ou faux (une pensée) —, mais à un objet spécifié, au moyen de la forme de nominalisation, en des termes qui impliquent que sa capacité à être faux n'est pas actualisée. Cela lui suffit pour être un vérifacteur.

Dans Mind and World, j'ai insisté sur le fait que l'expérience perceptuelle des sujets rationnels a un contenu qui est conceptuel sur toute la ligne. Dokic suit John Campbell en pensant qu'on peut pertinemment s'opposer à une telle conception sous prétexte que cela nous empêcherait de dire de l'expérience qu'elle explique la possibilité de saisir des pensées. Mais si les mêmes capacités qui sont exercées dans les jugements perceptuels sont aussi actualisées dans les expériences perceptuelles, cela nous aide à rendre intelligible l'intentionnalité, l'orientation vers le monde qu'exemplifient tous les deux types d'actualisation de telles capacités. L'explication que j'envisage ici ne procède pas dans la direction inverse à celle de l'explication suggérée par Campbell; je ne propose pas que l'intentionnalité du jugement soit intelligible de manière indépendante, de sorte qu'elle pourrait être invoquée pour expliquer l'intentionnalité de l'expérience perceptuelle. Mon idée, c'est que l'expérience perceptuelle et le jugement étant tous deux présents dans le portrait de la façon dont les capacités en question opèrent, on ne peut ici se

3. Dans le même contexte, Johnston parle de choses qui pourraient être fausses, comme si cette idée était interchangeable avec celle de choses qui auraient pu être fausses. Il peut être désinvolte sur cette question, sans doute, parce que son intérêt ne vise pas une spécification de la «réalité concrète » sur le modèle de la spécification du monde comme tout ce qui est le cas; il veut indiquer des objets comme des chaises et des instances de la propriété de dureté, qui ne sont aucunement porteurs de valeur de vérité, peu importe sous quelle modalité. Mais il est absolument erroné de penser que concevoir la réalité concrète comme tout ce qui est (concrètement) le cas nous empêche de trouver par exemple des chaises et des instances de dureté dans la réalité concrète - un peu comme l'image tractarienne nous permet de trouver des objets dans le monde, mais pas en tant que constituants. 
dire surpris quant à la question de savoir comment les opérations de ces capacités peuvent être dirigées vers le monde. Ni Campbell ni Dokic, qui le suit sur ce point, n'envisagent comment cette manière non directionnelle par laquelle une réflexion dont les thèmes incluent l'expérience peut contribuer à une compréhension de l'intentionnalité.

D'aucuns se sont opposés à l'affirmation selon laquelle le contenu expérientiel est conceptuel de part en part sous prétexte que le contenu conceptuel ne pourrait pas être individué aussi finement que le contenu de l'expérience. Dans Mind and World, j'ai répondu à cette objection en invoquant les concepts déictiques. Relativement à cela, Dokic affirme que tout concept pouvant être exprimé par «coloré de cette manière " - dont on pourrait penser qu'il est à la disposition d'un sujet en vertu, chez ce dernier, de la présence visuelle d'une qualité chromatique déterminée - serait "nécessairement moins spécifique» que la qualité chromatique présente dans l'expérience pertinente du sujet. Cela m'apparaît n'avoir rien qu'un semblant de cohérence. Dokic pense que le contenu conceptuel qui peut être exprimé en disant "cette chose est colorée de cette manière " (lorsque nous avons une expérience visuelle appropriée) mènerait à une disjonction: ou bien la chose vue a la qualité chromatique qui est visuellement présentée dans l'expérience en question, ou bien elle a une qualité chromatique que le sujet serait incapable de discerner de cette qualité même. Mais le second terme de la disjonction peut sembler nécessaire seulement si la qualité chromatique qui est visuellement présentée au sujet dans l'expérience en question, qui figure dans le premier terme de la disjonction, a une détermination dépassant la capacité du sujet de discerner les couleurs. Et comment cela se pourrait-il ? Comme l'indique Dokic, la notion d'une nuance de RussellGoodman est théorique. Mais alors, comment cela pourrait-il être une nuance de Russell-Goodman qui est présentée visuellement dans une expérience? Qu'une qualité chromatique nous soit présentée n'est pas comparable à avoir dans notre champ de vision quelqu'un qui est directeur de banque, par exemple. Dans ce cas, il n'est pas surprenant que notre expérience visuelle ne puisse pas nous fournir la connaissance qu'il est directeur de banque. Il est clair et certain que ce qui est présenté dans une expérience visuelle quant à la qualité chromatique d'une chose ne peut pas être plus déterminé que ce que l'expérience permettrait de connaître quant à la qualité chromatique de cette chose. Peut-être la conception des vérifacteurs de Dokic donne-t-elle l'impression qu'une telle divergence fait sens. Mais si c'est le cas, alors tant pis pour la conception des vérifacteurs de Dokic.

\section{Réponse à Pascal Engel}

Il y a une ambiguité que je regrette dans le premier passage cité par Engel dans lequel j'essaie d'exploiter la suggestion de Gareth Evans selon laquelle, lorsque nous parlons de sujets rationnels, nous devrions réserver le terme de «croyance» à un état cognitif sophistiqué à définir dans les termes de la 
notion de jugement et à comprendre d'une manière qui le lie à la notion de raisons.

Ce que je suggère dans ce passage quant à la manière de définir la croyance en termes de jugement, comme le propose Evans, c'est qu'une croyance, dans le sens pertinent, est une disposition à émettre des jugements. Or on peut concevoir l'acte de juger que telle-et-telle chose est le cas, tel que figuré dans la définition, comme une contrepartie non manifeste à affirmer, à voix haute, que telle-et-telle chose est le cas. D'une autre manière, nous pouvons peut-être concevoir l'affirmation en tant que telle comme un acte de juger à voix haute, auquel cas le juger simpliciter serait à l'assertion ce qu'est le genre à l'espèce, et non une contrepartie à une contrepartie. Des deux manières, on peut dire que le juger dans un sens comme celui-ci, dans lequel le juger est la manifestation d'une croyance comprise comme disposition, est une expression, manifeste ou interne, de la manière dont nous nous sommes (déjà) décidés.

Toutefois, ce à quoi je voulais vraiment faire appel, c'est à l'acte de juger que telle-et-telle-chose est le cas pris au sens de décider [making up one's mind] que telle-et-telle-chose est le cas. J'ai considéré que, pour désigner l'acte de décider, l'expression "acte de spontanéité » était de toute évidence appropriée. Effectivement, je pense que la désignation "acte de spontanéité " est appropriée de manière tout aussi évidente, pour les performances, manifestes ou internes, qui expriment comment on s'est (déjà) décidé et, bien que de manière moins évidente, aussi pour le fait de s'être décidé d'une certaine manière, mais je reviendrai là-dessus. Toutefois, c'est à l'acte de décider que je voulais faire référence pour introduire une notion intuitive de spontanéité dans le tableau. Dans le sens sur lequel j'aurais dû mettre l'accent — qui est en jeu dans le passage cité par Engel - juger que telle-et-telle-chose est le cas est un acte qui complète un type familier d'activité intellectuelle. En prenant part à cette activité, on est en processus de déterminer quoi penser à propos d'une certaine question, et, dans l'acte qui complète l'activité, on détermine (ici, le verbe a un aspect perfectif) quoi penser sur la question. L'acte de juger pris comme décision est en contraste avec l'acte de juger comme manifestation d'une croyance comprise comme disposition, juger comme étant une contrepartie peut-être non manifeste à dire ce qu'on pense déjà sur une question.

Comme je l'ai dit, je croyais que l'étiquette «acte de spontanéité » était évidemment appropriée pour ce type familier d'occurrence ce que je viens de décrire comme l'exercice d'un type d'activité intellectuelle. Cependant, Engel ne trouve pas évident que même s'ouvrir à la force des raisons, comme on le fait dans le type de processus qui culmine dans ce que j'appelle "se décider ", soit à compter comme une activité, ni (a fortiori) que son accomplissement devrait compter comme un acte de spontanéité.

Les questions d'Engel mettent en évidence le fait qu'un soin particulier est nécessaire à l'interprétation de l'idée que j'ai tenté d'exploiter afin 
d'introduire la notion de spontanéité: l'idée qu'un sujet rationnel contrôle la forme de sa pensée, d'une manière qu'on a très bien en vue (c'est du moins ce que je pensais) lorsqu'on détermine ce qu'on doit penser à propos d'une question.

Comme le note Engel, mon idée n'est pas qu'on peut insérer des croyances à volonté dans son esprit. En déterminant quoi penser à propos d'une question, on s'ouvre à la force des raisons qui portent sur la question. Idéalement, cela devrait culminer dans une compréhension de la croyance spécifique que les raisons nous enjoignent à avoir et l'acquisition de cette croyance. À aucun moment nous n'avons le choix d'avoir cette croyance - ni avant de comprendre quelle croyance les raisons nous enjoignent à avoir (et dans ce cas, avoir cette croyance n'est pas une option rationnelle), ni au moment où on en vient à comprendre quelle croyance les raisons enjoignent (dans ce cas nous n'avons aucune autre option rationnelle).

Engel pense que les considérations de ce genre indiquent qu'il n'y a aucun sens dans lequel un sujet peut compter comme actif en répondant aux exigences de la raison. Il mentionne la distinction de Pamela Hieronymi entre le contrôle évaluatif et le contrôle manipulatif. On est en contrôle évaluatif de sa pensée dans la mesure où ce qu'on pense est déterminé par la force des raisons qui ont trait à la question: quoi penser ? et c'est là le type de contrôle que je recherche. Mais Engel trouve déroutante la question de savoir comment cela peut être considéré comme un type de contrôle tout court.

Je peux proposer le début d'une réponse à cette question en considérant l'argument d'Engel selon lequel on est tout sauf actif, comme il le dit, lorsqu'on permet à nos croyances d'être déterminées par la force normative des raisons. J'ai reconnu qu'on n'opte pas pour l'une ou l'autre des croyances. Mais il serait absurde de considérer cela comme un motif pour nier la responsabilité de penser ce que l'on pense. Ce serait absurde de dire: "Je n'ai pu m'empêcher de former cette croyance; j'ai succombé à la force des raisons» - comme si avoir des croyances déterminées par les raisons revenait à avoir des croyances déterminées par des publicités astucieuses ou un lavage de cerveau. Au contraire: avoir des croyances déterminées par les raisons reposant sur leur vérité, c'est précisément être auto-déterminé dans ce que l'on pense.

L'idée de contrôle peut aisément nous mener à l'idée d'une capacité à manipuler ce dont on dit avoir le contrôle. C'est la seule idée de contrôle qui semble figurer dans le portrait suggéré par Engel. Dans ce portrait, le rôle de la conscience de soi rationnelle - qu'il conçoit comme un plus par rapport à la capacité d'avoir des croyances - est qu'elle nous permet de tenter de modifier nos croyances de premier degré lorsqu'on reconnaît des conflits entre ce qu'on croit et ce que l'on pense qu'on doit croire. Mais l'auto-détermination dans ce qu'on pense, comme je conçois la chose, est tout simplement absente dans ce portrait. S'apercevoir de quelle croyance les raisons nous enjoignent à avoir - ce que nous sommes tenus de croire 
rationellement - ne revient pas, comme dans le portrait d'Engel, à se placer dans une position où on a un motif pour tenter de provoquer le fait que nous avons cette croyance. Les cas pathologiques mis à part, il n'y a aucun fossé entre le fait de voir quelle croyance la raison nous enjoint à avoir et le fait d'en venir à avoir cette croyance. Il n'y a pas besoin d'une étape supplémentaire, qui pourrait ou non réussir, pour se persuader d'avoir cette croyance.

Il est peut-être inapproprié de considérer la formation de croyances en réponse aux raisons qui reposent sur la vérité de celles-ci comme étant l'exercice d'un type de contrôle sur ce qu'on pense. Mais il n'est pas nécessaire de se disputer sur le mot "contrôle». Le point essentiel est celui-ci : que notre pensée soit déterminée par des raisons qui reposent sur la vérité des réponses à nos questions est tout à fait l'inverse d'abdiquer à une force étrangère la détermination de ce que l'on pense. Comme je l'ai dit, voilà ce qu'est être auto-déterminé dans ce qu'on pense.

Dans le portrait que je dresse, la place de la conscience de soi rationnelle n'est pas qu'elle ajoute à l'ensemble des attitudes cognitives - qui seraient en tout cas en place - une capacité de jugement d'ordre supérieur concernant la position rationnelle des attitudes de premier ordre. C'est à propos d'un tel ajout supposé qu'Engel se demande comment celui-ci pourrait nous doter d'un contrôle sur les attitudes de premier ordre - et répond qu'il s'agit d'une condition nécessaire mais non suffisante à un tel contrôle, puisque nos efforts visant à avoir des croyances de premier ordre qui soient telles qu'on pense qu'elles doivent être pourraient ne pas réussir. (Le contrôle est considéré ici comme manipulatif.) Dans le portrait que je dresse, la conscience de soi rationnelle n'est pas un ajout à la capacité à croire dans le sens en question. Il est intrinsèque aux capacités, qu'on exerce lorsqu'on détermine quoi penser - dans des occurrences d'un genre qui, en tant qu'exercice de l'activité intellectuelle pris dans un sens parfaitement intuitif, garantissent pleinement la désignation "acte de spontanéité " et leurs actualisations sont à la portée de la conscience de soi. Une autre façon d'exprimer cela est de dire qu'il est essentiel aux capacités qu'on exerce en décidant quoi penser qu'elles relèvent d'une faculté consciente spontanée. Des capacités de cette sorte, c'est-à-dire des capacités dont l'actualisation est consciemment rationnelle, sont également exercées dans l'expression de la façon dont nous nous sommes décidés. Cela est évident dans le cas du discours significatif, et le point s'applique aisément aux contreparties non manifestes des actes de langage significatifs. Et avoir une croyance, dans le sens de l'état cognitif plus sophistiqué qu'Evans voulait isoler - s'être décidé - est aussi une actualisation de telles capacités. Nous pouvons l'appeler "un acte de spontanéité ", "acte " voulant alors dire "actualisation ", pour traduire le fait que, dans l'acte de croire pris en ce sens, des capacités opèrent, dont il est essentiel qu'elles puissent être exercées dans un acte de décision, ce qui les caractérise comme relevant d'une faculté consciente spontanée. 
À propos de la capacité de juger de manière critique, Engel se demande: "Comment cette capacité peut-elle être déjà présente dans la perception à travers le fait qu'elle est conceptuelle si l'on doit la comprendre comme un développement supplémentaire de la capacité de juger à travers une pensée consciente? » À mon avis, il est erroné ici de parler de «développement supplémentaire». La capacité de jugement critique n'est rien d'autre que la capacité de juger; il ne saurait y avoir de capacité de juger, en aucun sens pertinent, à laquelle on devrait ajouter la capacité de conscience de soi critique. Quand j'ai affirmé que chez les sujets rationnels, l'expérience perceptuelle est conceptuelle, mon idée était précisement que dans une telle expérience, les capacités qui sont des éléments de la faculté de juger de manière critique entrent en jeu. Bien sûr, d'aucuns ont trouvé cette affirmation incroyable. Toutefois, la question d'Engel n'est pas une simple expression d'incrédulité mais vise à indiquer une difficulté spécifique quant à l'affirmation. Pourtant, la difficulté supposée semble dépendre essentiellement de l'idée que la conscience de soi critique est un ajout à la capacité de croyance et de jugement. Que cette idée doive être rejetée, c'était là une partie du tableau que j'essayais de dresser ${ }^{4}$.

Traduit de l'anglais par Guillaume Fréchette (traduction revue par l'auteur)

4. Dans la préparation de cette réponse, j'ai été aidé par l'article encore non publié de Matthew Boyle intitulé "'Making up your mind' and the Metaphysics of Belief ", tout particulièrement en ce qui concerne l'idée que la conscience de soi critique est un ajout à une capacité autonome de croire et de juger. 